\title{
Cyclooxygenase inhibition attenuates endotoxin-induced spatial learning deficits, but not an endotoxin-induced blockade of long-term potentiation
}

\author{
Kendra N. Shaw ${ }^{\mathrm{a}}$, Sean Commins ${ }^{\mathrm{b}, *}$, Shane M. O'Mara ${ }^{\mathrm{a}}$ \\ ${ }^{a}$ Department of Psychology and Trinity College Institute of Neuroscience, Trinity College, Dublin 2, Ireland \\ ${ }^{\mathrm{b}}$ Department of Psychology, NUI, Maynooth, Maynooth, Co. Kildare, Ireland
}

Accepted 11 January 2005

\begin{abstract}
Peripheral administration of lipopolysaccharide (LPS), a potent bacterial endotoxin, can cause a variety of central effects, including production of cytokines and cyclooxygenases in the brain, as well as peripheral increases in corticosterone. These, in turn, may contribute to neuroimmune-induced neurocognitive deficits. We show here LPS causes deficits in hippocampal-dependent spatial learning in the water maze but that treatment with ibuprofen, a broad-spectrum cyclooxygenase inhibitor, reverses the deficits induced in spatial learning by LPS. We also show that LPS causes an impairment in the induction of long-term potentiation in the dentate gyrus in vivo, a major contemporary model of learning and memory. No differences were found in corticosterone levels in trunk blood but we find a decrease in brain-derived neurotrophic factor (BDNF) expression in LPS group compared to saline controls. Paradoxically compared to the behavioral findings treatment with ibuprofen does not attenuate the LPS-induced impairment in LTP or BDNF concentration in tetanized tissue.
\end{abstract}

(C) 2005 Elsevier B.V. All rights reserved.

Theme: Endotoxins

Topic: Synaptic plasticity and learning

Keywords: Cyclooxygenase inhibition; Lipopolysaccharide; Long-term potentiation

\section{Introduction}

Lipopolysaccharide (LPS), a potent bacterial endotoxin, produces a wide range of non-specific behavioral effects collectively termed 'sickness behaviors' [18]. Such behaviors include a reduction in activity and exploration, decreased social interaction, fever, a reduction in consumption of food and drink, hypersomnia, activation of the hypothalamic-pituitary-adrenal axis and increased sympathetic activation [15,24,27]. The exact mechanism of action by LPS in the central nervous system (CNS) remains unclear; it was previously thought to act primarily through

\footnotetext{
* Corresponding author. Fax: +353 17084767.

E-mail address: Sean.Commins@may.ie (S.Commins).
}

the release of cytokines [10], which activate a chain of immune and endocrine responses [11], including increases in corticosterone. Now, evidence suggests that LPS activates Toll-like receptor 4 (TLR-4) in the circumventricular organs and result in a NFKappaB-dependent induction of proinflammatory cytokines [19].

The pro-inflammatory enzyme cyclooxygenase is found in two isoforms in neurons (COX1 and 2); LPS induces the expression of $\mathrm{COX} 2$ and leads to the production of prostaglandins (PGs) $[24,32]$. Inhibition of the COX pathway of arachidonic acid (AA) metabolism has been the accepted explanation for the anti-inflammatory, analgesic, and antipyretic properties of non-steroidal anti-inflammatory drugs (NSAIDs) [8]. Both COX isoforms are expressed in hippocampal neurons $[4,31]$. 
The hippocampal formation plays a critical role in certain types of learning and memory [30]. Recent evidence suggests that LPS may disrupt certain mnemonic processes: acute administration of LPS prior to training impairs contextual fear conditioning, a hippocampal-dependent learning paradigm [27], while a chronic infusion of LPS has been found to impair spatial memory [16,17]. Furthermore, administration of IL-1 beta impairs performance of rats in the Morris water maze, another hippocampaldependent learning task [25]. Long-term potentiation (LTP) of synaptic transmission is a popular model of the biological processes that may underlie memory $[2,3]$. LTP is readily induced in the hippocampus; blocking LTP causes impairment on the water maze task [23]. LPS inhibits LTP in the rat dentate gyrus in vitro [9] and in the CA1subiculum pathway in vivo [7].

We have recently shown that a broad-spectrum cyclooxygenase inhibitor, ibuprofen (IBU), can affect both synaptic plasticity and hippocampal-dependent learning and that these deficits can be reversed by increasing endogenous brain-derived neurotrophic factor (BDNF, [29]). BDNF plays a critical role in the survival and growth of neurons. High levels of this neurotrophin are found in the hippocampus and have been implicated in hippocampal-dependent learning. Recent studies have found that BDNF also regulates synaptic plasticity; more specifically, the exogenous application of BDNF enhances synaptic transmission in hippocampal neurons and enhances LTP [19]. BDNF expression is increased in the hippocampus during spatial learning tasks, lending further support for its role in learning and memory [13,14]. Little research has been performed on the effects of LPS on BDNF expression, and the existing research is conflicting. Elkabes et al. [12] found BDNF expression was unaltered in the rat brain in the presence of LPS, whereas Miwa et al. [22] found that LPS stimulated the synthesis of BDNF. Shaw et al. [28] injected rats with LPS intraperitoneally and trained them in the water maze. A single injection of LPS impaired escape latency in both the acquisition and retention phases of the study, whereas a daily injection of LPS did not significantly impair acquisition or retention. No significant differences in BDNF expression were found between the three groups. LPS, by triggering cytokine expression, may contribute to neuropathological cell death via COX expression. It follows that by blocking the LPS-induced COX expression, there should be a reversal of the observed LPS-produced learning deficit.

The experiments presented here examine the effects of LPS on hippocampal-dependent spatial learning, LTP and BNDF expression in the dentate gyrus of the hippocampal formation. We also investigate the pharmaco-therapeutic effects of ibuprofen (a broad-spectrum COX inhibitor) [8] on LPS-treated animals. We hypothesize that ibuprofen will attenuate the LPS-induced learning deficit in both the water maze and LTP. We expect that by blocking LPS-induced
COX expression, that the LPS-produced learning deficit should be reversed.

\section{Methods and materials}

\subsection{Animals}

Adult male Wistar rats (BioResources Unit, University of Dublin; weight: $200-300 \mathrm{~g}$ ), pair-housed in a temperaturecontrolled laminar airflow cupboard, on a 12:12 light/dark cycle with free access to food and water, were used. Rats were pair-housed and maintained on a 12:12 light/dark cycle. Guideline for the maintenance and experimentation of animals conformed to the Department of Health (Ireland) guidelines and European directive 86/609/EC. Every effort was made to minimize the suffering and the number of animals used.

\subsection{Behavioral experiments}

Rats were randomly assigned to one of 4 groups $(n=$ 6/group):

(1) Single LPS injection: LPS (i.p. $250 \mu \mathrm{g} / \mathrm{kg}$ obtained from Escherichia coli; (L-2630; $100 \mathrm{mg}$ ) Sigma, USA) injected once on the first day of the acquisition phase $4 \mathrm{~h}$ before training.

(2) LPS and ibuprofen co-administration injection: Animals were pre-treated with ibuprofen (i.p. $30 \mathrm{mg} / \mathrm{kg}$ obtained from Sigma, USA) $1 \mathrm{~h}$ before the LPS (i.p. $250 \mu \mathrm{g} / \mathrm{kg}$ ) injection; animals were tested $4 \mathrm{~h}$ after the LPS injection.

(3) Saline control: Saline (i.p. 0.9\%) injected once on the first day of the acquisition phase $4 \mathrm{~h}$ before training.

(4) Ibuprofen-only: Ibuprofen (i.p. $30 \mathrm{mg} / \mathrm{kg}$ obtained from Sigma, USA) was injected once on the first day of the acquisition phase $1 \mathrm{~h}$ before training.

\subsubsection{Water maze training}

All water maze experiments were conducted using an automated computerized digital tracking system (EthoVision, Noldus, Netherlands), using standardized protocols [28]. The water maze was a black circular pool $(2 \mathrm{~m}$ diameter, $35 \mathrm{~cm}$ deep; water, $20 \pm 1{ }^{\circ} \mathrm{C}$ ) filled to $31 \mathrm{~cm}$. Rats could escape the water by climbing on to a hidden platform $(29 \mathrm{~cm} \times 9 \mathrm{~cm})$. The hidden platform was placed in the northeastern quadrant of the pool and submerged 2 $\mathrm{cm}$ below the water surface so it was invisible at water level; the location of the platform was fixed during the experiment. Distal cues were standard room objects (e.g., doors, shelving, and curtains). Rats received five trials per day for 5 days, where they had to search for the hidden platform. The rat was allowed $60 \mathrm{~s}$ to find the platform; otherwise, the rat was led to the platform by the researcher. 
The rat remained on the platform for $15 \mathrm{~s}$. The inter-trial interval was $5 \mathrm{~s}$. Following the 5 days training, animals were allowed to rest for a further 3 days. Retention was examined by a further 3 days of trials.

\subsection{Electrophysiological procedures}

Rats were randomly assigned to one of 4 groups ( $n=$ 6/group).

(1) Single LPS injection: LPS (i.p. $250 \mu \mathrm{g} / \mathrm{kg}$ obtained from Escherichia coli; (L-2630 $100 \mathrm{mg}$ ): Sigma Chemical, St. Louis, MO, USA) injected $4 \mathrm{~h}$ before anesthesia.

(2) LPS and ibuprofen co-administration injection: Animals were pre-treated with ibuprofen (i.p. $30 \mathrm{mg} / \mathrm{kg}$ obtained from Sigma, USA) $1 \mathrm{~h}$ before the LPS (i.p. $250 \mu \mathrm{g} / \mathrm{kg}$ ) injection; animals were anesthetized $4 \mathrm{~h}$ after the LPS injection.

(3) Saline control: Saline (i.p. $0.9 \%$ ) was injected $1 \mathrm{~h}$ before anesthesia.

(4) Animals were pre-treated with ibuprofen (i.p. $30 \mathrm{mg} /$ $\mathrm{kg}$ obtained from Sigma, USA) $4 \mathrm{~h}$ before anesthesia.

\subsubsection{Electrophysiology}

All electrophysiological experiments were conducted in vivo (see Ref. [5] for details). In brief stimulating electrodes (8.1 posterior to Bregma and $4.4 \mathrm{~mm}$ lateral) were aimed at the perforant path and recording electrodes (3.9 $\mathrm{mm}$ posterior to Bregma and $2.5 \mathrm{~mm}$ lateral) at DG [26]. Signals were filtered $(0.1 \mathrm{~Hz}-1 \mathrm{kHz})$ and amplified $(\times 100$; DAM-50 differential amplifier, WPI, UK). Recordings were digitized online using a PC connected to a CED1401 interface (CED, UK). Electrodes were slowly lowered to a depth of $2.5 \mathrm{~mm}$; test stimuli were administered during electrode movement at $0.05 \mathrm{~Hz}$. The final depths were adjusted until maximal fEPSPs were obtained; electrodes were allowed to settle for $10 \mathrm{~min}$ before recordings were conducted at half-maximum fEPSPs (using individually determined input-output curves). Field EPSPs are expressed as percent baseline slope (mean \pm SEM).

LTP was induced by high-frequency stimulation (HFS: 10 trains of 20 stimuli at $200 \mathrm{~Hz}$, inter-train interval of $2 \mathrm{~s}$; [6]), after which baseline stimulation was resumed and fEPSPs recorded for $30 \mathrm{~min}$.

\subsection{Measurement of BDNF and blood corticosterone}

After each experiment, the animals were stunned, decapitated quickly and the brain was removed. The hippocampus was dissected out bilaterally according to standard methods $[13,14,28]$ and the DG was isolated, chopped and stored $\left(-70{ }^{\circ} \mathrm{C}\right)$ pending subsequent biochemical analysis. Concentrations of BDNF were determined using ELISA.
2.4.1. BDNF enzyme-linked immunosorbent assay protocol (BDNF Emax ${ }^{\mathrm{TM}}$ ImmunoAssay System; Promega, UK, Ltd.)

Slices of dentate gyrus were preincubated in $250 \mu \mathrm{l}$ Krebs solution containing $2 \mathrm{mM} \mathrm{CaCl}{ }_{2}$ for $3 \mathrm{~min}$, and the supernatant was removed and discarded. This step was repeated in a volume of $100 \mu \mathrm{l}$, and at the end of the 3-min incubation samples were centrifuged at $1000 \times g$ for $3 \mathrm{~min}$ and the supernatant was retained. This step was repeated in the presence of $40 \mathrm{mM} \mathrm{KCl}$ to depolarize the slices and supernatant was retained. Both samples of supernatant were stored at $-70{ }^{\circ} \mathrm{C}$ for later analysis of BDNF by two-site immunoassay. BDNF concentration was determined in the supernatant. Dentate gyri were homogenized in ice-cold Krebs solution 25 times, centrifuged and the supernatant retained. Protein was assessed and samples were diluted to give equal protein concentrations and stored at $-80{ }^{\circ} \mathrm{C}$. Ninety-six well plates were coated with $100 \mu \mathrm{l}$ anti-BDNF monoclonal antibody diluted (1:1000) in $0.025 \mathrm{M}$ carbonate-bicarbonate buffer. Plates were covered, incubated overnight at $4{ }^{\circ} \mathrm{C}$ and plates were subjected to interceding washes to remove excess antibody. Plates were blocked for non-specific binding for $1 \mathrm{~h}$ at room temperature and washed (composition of wash buffer (mM): Tris- $\mathrm{HCl}, 20 ; \mathrm{NaCl}, 150$ containing $0.05 \%$ Tween (v/v); pH 7.6). Samples of dentate gyrus $(50 \mu \mathrm{l})$, supernatant $(50 \mu \mathrm{l})$ or BDNF standards $(50 \mu \mathrm{l}$; ranging from 0.0078 to $1 \mathrm{ng} / \mathrm{ml}$ ) were added to the wells, which were then covered, incubated for $2 \mathrm{~h}$ at room temperature with shaking, and then incubated overnight at $4{ }^{\circ} \mathrm{C}$, and washed. Aliquots $(100 \mu \mathrm{l})$ of anti-human BDNF pAb (diluted 1:500) were added to the wells, plates were incubated for $2 \mathrm{~h}$ at $37{ }^{\circ} \mathrm{C}$ and washed. Aliquots $(100 \mu \mathrm{l})$ of anti-immunoglobulin horseradish peroxidase (1:2000 dilution) were added to wells and incubated for $1 \mathrm{~h}$ at $37{ }^{\circ} \mathrm{C}$. During this incubation, the enzyme substrate was prepared. Plates were washed and $100 \mu$ of this substrate was added to the wells, and incubated for approximately 15 min until a blue color formed in the wells. The reaction was stopped by the addition of $100 \mu \mathrm{l}$ of $1 \mathrm{M}$ phosphoric acid to the wells. Plates were read a $450 \mathrm{~nm}$ in a 96-well plate reader, and BDNF concentrations were estimated for the standard curve (expressed as ng/mg protein).

Corticosterone levels were measured by radioimmunoassay in trunk blood. The serum was collected on decapitation and then rapidly frozen at $-20{ }^{\circ} \mathrm{C}$ and stored for subsequent analysis (DPC, USA; [29]). With this procedure serum samples are assayed directly, without preparation. Samples were thawed to room temperature and $50 \mu \mathrm{L}$ of either serum or calibrator (ranging from 0 to $2000 \mathrm{ng} / \mathrm{mL}$ corticosterone) was added to the rat corticosterone $\mathrm{AB}$ Coated test tubes. These tubes were pre-coated with antibodies to rat corticosterone. $100 \mathrm{~mL}$ of ${ }^{125} \mathrm{I}$ Rat Corticosterone was added to every tube and incubated for $2 \mathrm{~h}$ at room temperature. The tubes were then decanted and counted for 1 min in a gamma counter. Concentrations were estimated from 
the calibrator standard curve and expressed as $\mathrm{ng} / \mathrm{mL}$ of corticosterone.

\subsection{Statistics}

Behavioral data were analysed using repeated measures ANOVA; BDNF ELISA values and corticosterone levels were analysed using a one-way ANOVA; a value of $P<$ 0.05 was considered to be significant. Between groups post hoc comparisons were made using Tukey's method $(P<$ $0.05)$. Dependent $t$ tests were used to analyze the electrophysiological data within group and ANOVA were used to compare across groups.

\section{Results}

\subsection{Water maze training and retention analysis}

\subsubsection{Direct Swim analysis}

A direct swim pathway to the hidden platform was defined (see Ref. [28], this is thought to be a more stringent measure of learning). The percentage time animals spent in this pathway $(30 \mathrm{~cm}$ wide from the start position to the platform, see Fig. 1a inset) was used to measure the most direct route. Overall main effects within groups were found (Days 1-5: $F(4,384)=46.993, P<$ 0.001; Days 9-11: $F(2,160)=11.42, P<0.001)$; post hoc testing did not show any significant differences across days suggesting that all 4 groups were able to learn the water maze task. However, further analysis revealed that the LPS group and the ibuprofen group failed to use the most direct route to find the hidden platform compared to the other groups. On day 1, the LPS group was significantly worse than the LPS and ibuprofen groups $(P<0.01)$; both the saline controls and the LPS and ibuprofen used the most direct route to find the platform on day 4 compared to the LPS and ibuprofen-only groups. Fig. 1b shows representative swim paths taken from each group on day 5. Similarly, the LPS-only group did not take the most direct route to the platform during the retention phase of the task. In addition, no significant difference in dentate BDNF concentration or blood corticosterone concentration was found between the groups on the final day (day 11; data not shown).

\subsection{Electrophysiological results}

\subsubsection{Effect of LPS, LPS and ibuprofen and saline on synaptic plasticity}

In saline-treated animals, LTP was successfully induced. Field EPSPs remained potentiated for at least $30 \mathrm{~min}$ as indicated by the change in fEPSP slope which stood at $139.27 \pm 10.8 \%, 146.39 \pm 9.3 \%, 150.5 \pm 9.5 \%$ of baseline at 5, 15 and $30 \mathrm{~min}$ post-HFS, respectively (see Fig. 2). The mean percentage increase in fEPSP response for the final $10 \mathrm{~min}$ of the recording period stood at $110 \pm$ $0.44 \%, 111 \pm 0.58 \%$ and $103.7 \pm 0.41 \%$ for the LPS, LPS and ibuprofen, and ibuprofen-only groups, respectively. An overall significant difference was found between the four groups for last $10 \mathrm{~min}$ of the recording period (ANOVA: $F(3,39)=53.095, P<0.001)$. Post hoc tests showed that the mean increase of the fEPSP response of the saline group was significantly higher when compared to the other three groups.

BDNF concentration was determined in the dentate gyrus from all animals in each of the four groups. A $2 \times 4$ way ANOVA was used. Stimulation (stimulated Vs unstimu- a

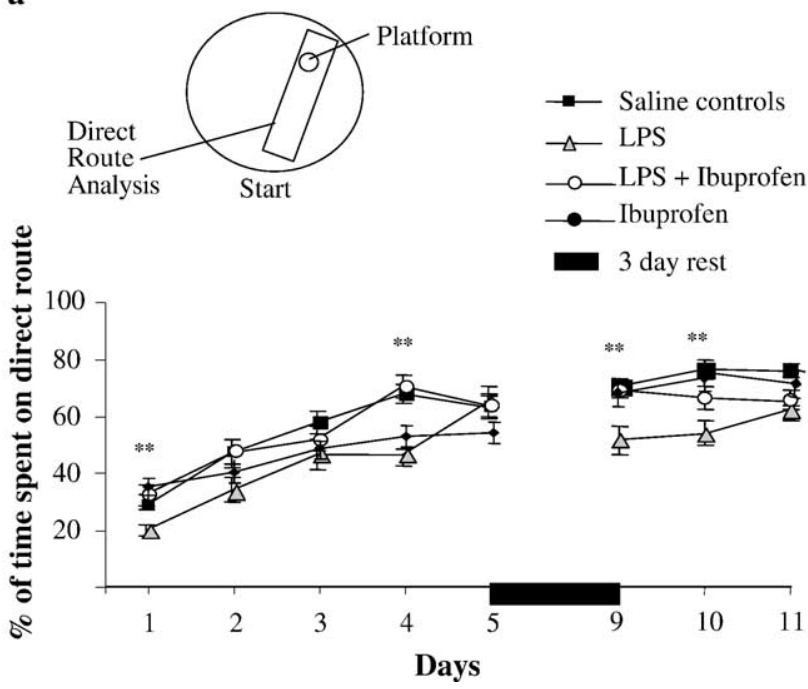

b

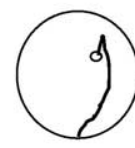

Control

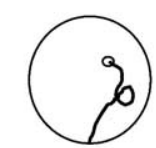

LPS + Ibuprofen

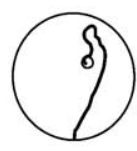

LPS

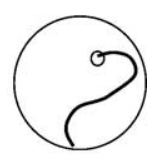

Ibuprofen
Day 5

Fig. 1. (a) Percentage of time spent in the direct route to the platform for all groups across the acquisition and retention periods. (b) Representative swim paths taken from the last days of acquisition (day 5) for each group $(n=6 /$ group; $* P<0.05$; ** $P<0.01)$. 


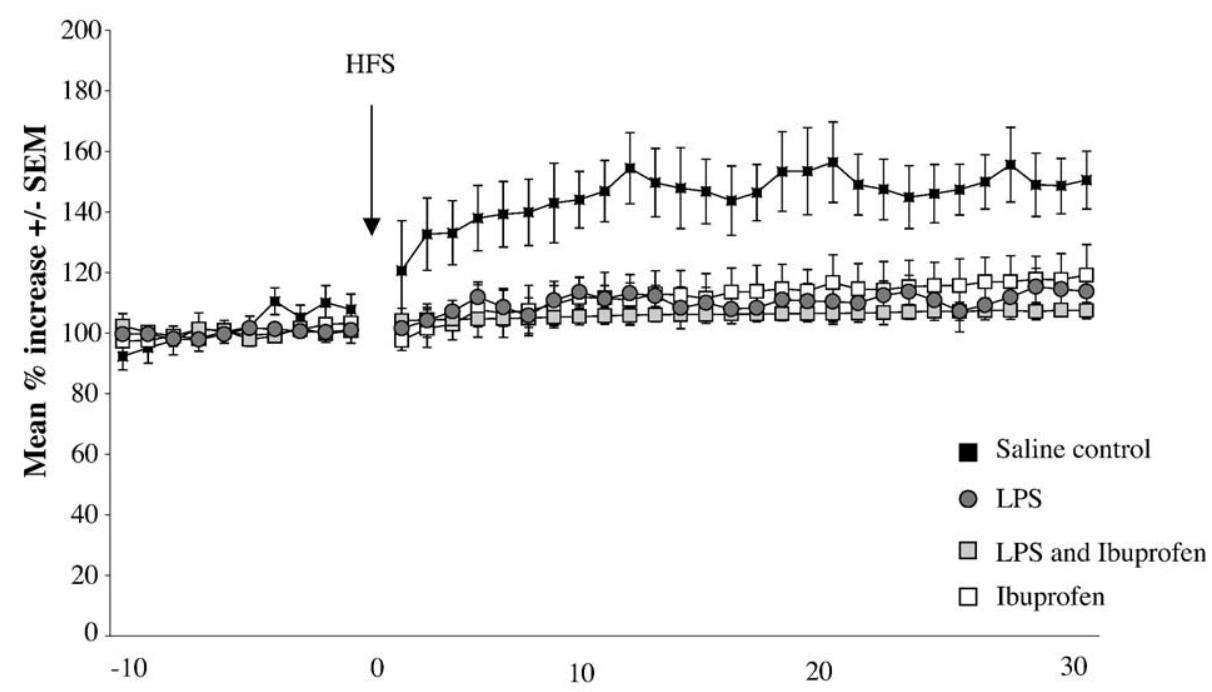

Fig. 2. Percentage change in amplitude of fEPSPs after HFS in all groups.

a

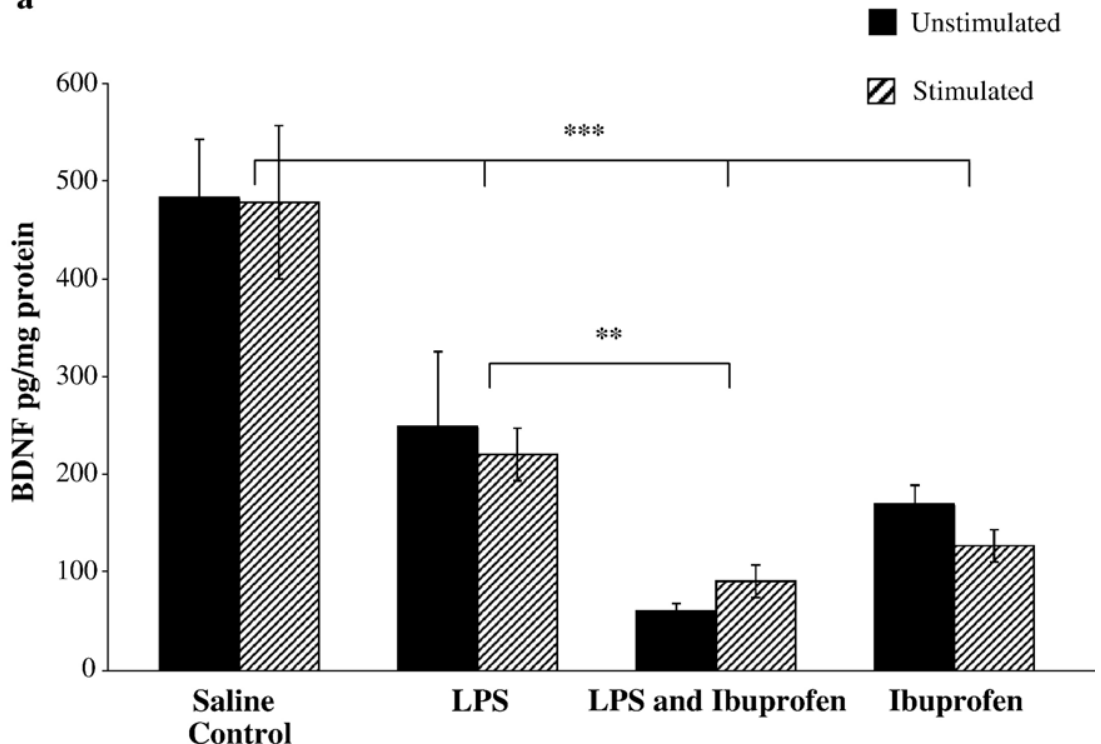

b

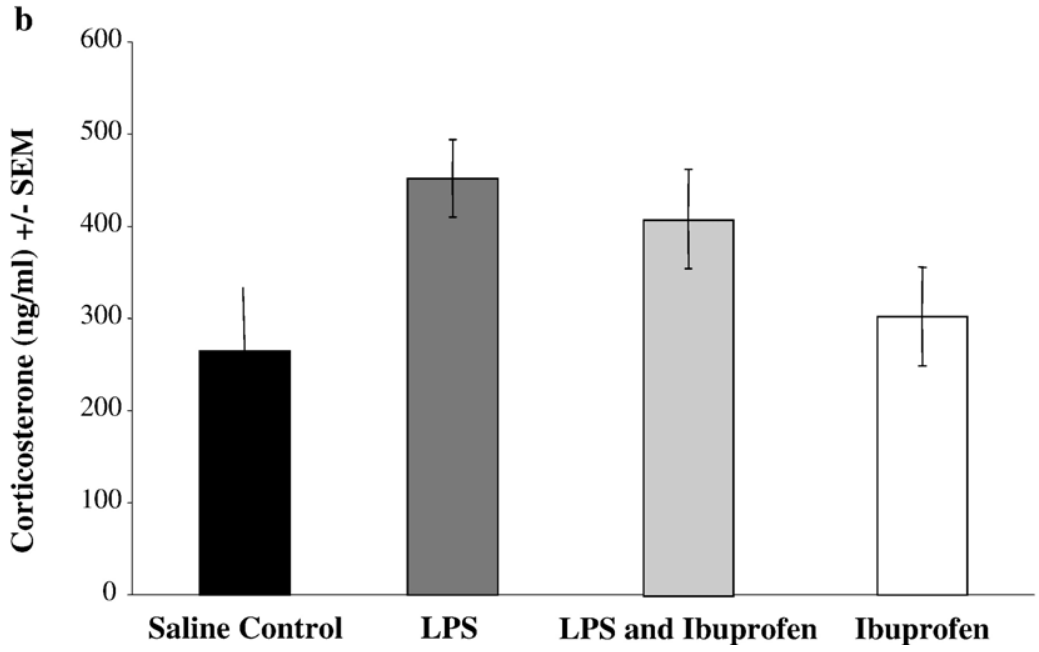

Fig. 3. (a) Mean concentration of BDNF in dentate gyrus from tetanized and untetanized tissue; (b) Average corticosterone levels from animals in the above experiment $(n=6 /$ group; $* P<0.05 ; * * P<0.01)$. 
lated) was used as one between group condition and group (saline, LPS, LPS and Ibuprofen, and Ibuprofen-only) as the second between group condition. These analyses revealed a non-significant effect for stimulation $(F=0.85, d f=1.48$, $P>0.05)$, a non-significant interaction effect $(F=0.242$; $d f=3.48, P>0.05)$. However there was a significant effect for group (see Fig. 3a; $F=29.54, d f=3.48, P<0.001$ ). Subsequent post hoc analysis revealed that the saline group had significantly higher concentrations of BDNF and compared to the other three groups. In addition, the LPSonly group had significantly higher BDNF concentrations when compared to the LPS and ibuprofen group.

Corticosterone levels were determined from trunk blood taken immediately after recording. Fig. $3 b$ shows that no significant differences were found between the four groups (ANOVA: $F(3,19)=3.211, P>0.05)$.

\section{Discussion}

We investigated the neuroprotective effects of cyclooxygenase inhibition (using the broad-spectrum cyclooxygenase inhibitor, ibuprofen) in LPS-treated animals in relation to both spatial learning and LTP induction. We were particularly interested to investigate if ibuprofen attenuated the LPS-induced spatial learning and LTP blockade. We found that the LPS and ibuprofen co-treatment group learned the task better than the LPS-only group, as shown by the direct swim route analysis. We also found that there were no differences in BDNF concentration or corticosterone levels between the different treated groups and controls.

The set of experiments presented here demonstrate that peripheral stimulation by the endotoxin LPS impairs longterm potentiation in the dentate-gyrus in vivo. These findings replicate our own previous results in the CA1subiculum projection [7] and confirm the findings by Cunningham et al. [9] who found that LPS also blocks LTP in the dentate gyrus in vitro. We had suggested in the introduction that this LPS-induced blockade of LTP may result via an increase in COX expression. If this were the case, then co-administration of LPS and the COX inhibitor ibuprofen would attenuate the LPS-induced deficit in LTP. However, we found that this did not occur and that LPS and ibuprofen-treated group also impaired LTP induction. It is possible that LPS mediates its effect on synaptic plasticity via an alternative mechanism to that used during spatial learning and memory. It is also known that the inflammatory cytokines are expressed in the hippocampus during seizures and Alzheimer's disease [1] suggesting that these cytokines affect normal hippocampal functioning both at the cellular and behavioral level. In addition to this, IL-1 beta has been shown to inhibit the induction of LTP in areas CA1 and CA3 of the hippocampus but does not impair spatial navigation [25]. An alternative explanation of our findings may be accounted by a complicated interaction effect between COX, BDNF and synaptic plasticity. We show that the IBU-only group also seemed to cause a deficit in LTP, as seen previously (see Ref. [29]). Therefore, any effect of COX inhibition on the blockade of LTP by LPS may be masked by the deficit observed with the IBU group alone.

No differences were found in corticosterone levels between the groups, suggesting that the inhibition of LTP was not due to increased circulating glucocorticoids. Furthermore, we report that the saline-controls have increased BDNF levels compared to the other groups. We have previously shown that spatial learning increases BDNF concentration and others have found an increase in BDNF in tetanized tissue [14]. We, thus, would expect to find an increase in BDNF in the saline-controls. As LTP was inhibited in the other groups, we would not expect an increase in BDNF. Interestingly, there was a difference in BDNF concentration between the LPS-only group and the LPS and ibuprofen group perhaps giving credence to the idea of a complex interaction effect between the COX enzyme and neurotrophin expression.

There was no difference in BDNF concentration between the water maze-trained groups yet there was a difference between the tetanized groups. It appears that the BDNF increase that facilitates learning and/or LTP is time dependent. The tissue analysed from the water maze-trained animals was taken 11 days after the initial LPS and ibuprofen administration, whereas the tetanized dentate gyri were taken $6 \mathrm{~h}$ following LPS injection. The BDNF increase after HFS suggests that BDNF-dependent LTP is needed for the encoding of memory and once this process had finished, BDNF levels gradually return to baseline over a period of days. This explains the lack of BDNF differences in the spatially trained animals as, by day 11 , the memory had been encoded and consolidated, returning BDNF levels to baseline.

There is very little research investigating the interaction between COX regulation and neurotrophins. The stimulation of glutamate receptors by kainic acid increases COX2 levels in rat hippocampus [21]. Glutamate also stimulates NMDA receptors that permit an influx of $\mathrm{Ca}^{2+}$ into the post-synaptic cell. This $\mathrm{Ca}^{2+}$ influx stimulates a cascade of reactions required for LTP, one end product being BDNF transcription [2,14]. Manev et al. [21] reported a link between increased glutamate, $\mathrm{Ca}^{2+}$ and $\mathrm{COX} 2$ activation. This finding suggests a possible relationship between the Ras and phospholipase A2-arachidonic acid pathways. Furthermore, c-fos is rapidly induced by hippocampal NMDA activation and this NMDA response is blocked by a COX inhibitor [20]; this response must involve events in the nucleus, suggesting a COX-dependent signal linking receptor activation and gene expression. The stimulation of COX by LPS activates the COX-PG pathway thus reducing the available COX required for the stimulation of plastic related pathways. By inhibiting the LPS induced production of COX with a NSAID and thus blocking the 
stimulation of the AA-PG pathway, plasticity-dependent $\mathrm{Ca}^{2+}$ may stimulate constituent $\mathrm{COX}$ levels required for further changes.

\section{Acknowledgments}

European Commission and the Health Research Board of Ireland for financial support. We also thank Professor Marina Lynch (TCD) for helpful discussions.

\section{References}

[1] S.M. Allan, N.J. Rothwell, Cytokines and acute neurodegeneration, Nat. Rev., Neurosci. 2 (2001) 734-744.

[2] T. Bliss, T. Lomo, Long-lasting potentiation of synaptic transmission on the dentate area of the anaesthetised rabbit following stimulation of the perforant path, J. Physiol. 232 (2) (1973) 331-356.

[3] T.V.P. Bliss, G.L. Collingridge, A synaptic model of memory: longterm potentiation in the hippocampus, Nature 361 (6407) (1993) $31-39$.

[4] C.D. Breder, D. Dewitt, R.P. Krang, Characterization of inducible cyclooxygenase in rat brain, J. Physiol. 355 (2) (1995) 296-315.

[5] S. Commins, S.M. O'Mara, Interactions between paired-pulse facilitation, low-frequency stimulation and behavioural stress in the pathway from hippocampal area CA1 to the subiculum: dissociation of baseline synaptic transmission from paired-pulse facilitation and depression of the same pathway, Psychobiology 28 (1) (2000) 1-11.

[6] S. Commins, J. Gigg, M. Anderson, S.M. O'Mara, The projection from hippocampal area CA1 to the subiculum sustains long-term potentiation, NeuroReport 9 (1998) 847-850.

[7] S. Commins, L.A.J. O'Neill, S.M. O'Mara, The effects of the bacterial endotoxin lipopolysaccharide on synaptic transmission and plasticity in the CA1-subiculum pathway in vivo, Neuroscience 102 (2) (2001) $273-280$.

[8] L.J. Crofford, P.E. Lipsky, P. Brooks, S.T. Abramson, L.S. Simon, L.B.A. van de Putte, Basic biology and clinical applications of specific cyclooxygenase-2 inhibitors, Arthritis Rheum. 43 (1) (2000) 4-13.

[9] A.J. Cunningham, C.A. Murray, L.A.J. O’Neill, M.A. Lynch, J.J. O'Connor, Interleukin-1 beta and tumour necrosis factor inhibit longterm potentiation in the rat dentate gyrus in vitro, Neurosci. Lett. 203 (1) (1996) $17-20$

[10] S.K. Drum, J.J. Oppemtom, Macrophage derived mediators: IL1, TNF, IL6, IFN and related signals, in: W.F. Paul (Ed.), Fundamental Immunology, Raven Press, New York, 1989, pp. 639-661.

[11] A.J. Dunn, A. Wang, Cytokine effects on CNS biogenic amines, Neuroimmunomodulation 2 (6) (1995) 319-328.

[12] S. Elkabes, L. Peng, I.B. Black, Lipopolysaccharide differentially regulates microglial trk receptor and neurotrophin expression, J. Neurosci. Res. 54 (1) (1998) 117-122.

[13] M. Gooney, M.A. Lynch, Long-term potentiation in the dentate gyrus of the rat hippocampus is accompanied by brain-derived neurotrophic factor-induced activation of TrkB, J. Neurochem. 77 (5) (2001) 1198-1207.

[14] M. Gooney, K. Shaw, A. Kelly, S.M. O’Mara, M.A. Lynch, Long-term potentiation and spatial learning are associated with increased phosphorylation of TrkB and ERK in dentate gyrus: evidence for a role for BDNF, Behav. Neurosci. 116 (2002) 455-463.
[15] B.L. Hart, Biological basis of the behavior of sick animals, Neurosci. Biobehav. Rev. 12 (2) (1988) 123-137.

[16] B. Hauss-Wegrzyniak, P. Dobrzanski, J.D. Stoehr, G.L. Wenk, Chronic neuroinflammation in rats reproduces components of the neurobiology of Alzheimer's disease, Brain Res. 780 (2) (1998) 294-303.

[17] B. Hauss-Wegrzyniak, M.G. Vannucchi, G.L. Wenk, Behavioral and ultrastructural changes induced by chronic neuroinflammation in young rats, Brain Res. 859 (2000) 157-166.

[18] S.L. Klein, R.J. Nelson, Activation of the immune-endocrine system with lipopolysaccharide reduces affiliative behaviours in voles, Behav. Neurosci. 113 (5) (1999) 1042-1048.

[19] J.P. Konsman, P. Parnet, R. Dantzer, Cytokine-induced sickness behaviour: mechanisms and implications, Trends Neurosci. 25 (3) (2002) $154-159$.

[20] L.S. Lerea, J.O. McNamara, Ionotropic glutamate receptor subtypes activate $\mathrm{c}-f o s$ transcription by distinct calcium-requiring intracellular signaling pathways, Neuron 10 (1) (1993) 31-41.

[21] H. Manev, T. Uz, T. Qu, 5-Lipoxygenase and cyclooxygenase mRNA expression in rat hippocampus: early response to glutamate receptor activation by kainate, Exp. Gerontol. 35 (9-10) (2000) $1201-1209$

[22] T. Miwa, S. Furukawa, K. Nakajima, Y. Furukawa, S. Kohsaka, Lipopolysaccharide enhances synthesis of brain-derived neurotrophic factor in cultured rat microglia, J. Neurosci. Res. 50 (6) (1997) $1023-1029$

[23] RG.M. Morris, E. Anderson, G.S. Lynch, M. Baudry M, Selective impairment of learning and blockade of long-term potentiation by an $N$-methyl-D-aspartate receptor antagonist, AP5, Nature 319 (6056) (1986) 774-776.

[24] P. Needleman, Selective regulation of cellular cyclooxygenase by dexamethasone and endotoxin in mice, J. Clin. Invest. 86 (1990) $1375-1379$

[25] M.S. Oitzl, H. Van Oers, B. Schobitz, WR. de Kloet, Interleukin-1 beta, but not interleukin-6, impairs spatial navigation learning, Brain Res. 613 (1) (1993) 160-163.

[26] G. Paxinos, C. Watson, The Rat Brain in Stereotaxic Coordinates, Academic Press, San Diego, 1998.

[27] C.R. Pugh, K. Kumagawa, M. Fleshner, L.R. Watkins, S.F. Maier, J.R. Rudy, Selective effects of peripheral lipopolysaccharide administration on contextual and auditory-cue fear conditioning, Brain Behav. Immunol. 12 (3) (1998) 212-229.

[28] K.N. Shaw, S. Commins, S.M. O'Mara, Lipopolysaccharide causes deficits in spatial learning in the watermaze but not in BDNF expression in the rat dentate gyrus, Behav. Brain Res. 124 (1) (2001) $47-54$

[29] K.N. Shaw, S. Commins, S.M. O'Mara, Deficits in spatial learning and synaptic plasticity induced by the rapid and competitive broadspectrum cyclooxygenase inhibitor ibuprofen are reversed by increasing endogenous brain-derived neurotrophic factor, Eur. J. Neurosci. 17 (2003) 2438-2446.

[30] L. Squire, Memory and the hippocampus: a synthesis from findings with rats, monkeys and humans, Psychol. Rev. 99 (1992) v $195-231$.

[31] K. Yamagata, K.I. Andreasson, W.E. Kaufmann, C.A. Barnes, P.F. Worley, Expression of a mitogen-inducible cyclooxygenase in brain neurons: regulation by synaptic plasticity and glucocorticoids, Neuron 11 (2) (1993) 371-386.

[32] J. Zhang, S. Rivest, Anti-inflammatory effects of prostaglandin E2 in the central nervous system in response to brain injury and circulating lipopolysaccharide, J. Neurochem. 76 (3) (2001) 855-864. 